\title{
Evolución de la capacidad tecnológica en México. Aplicación del análisis estadístico multivariante de cluster
}

\author{
Evolution of state clusters related with technological capability \\ in Mexico: Application of a multivariate statistical analysis of cluster \\ Carla Carolina Pérez Hernández*, Graciela Lara Gómez \\ y Denise Gómez Hernández
}

Universidad Autónoma de Querétaro, México

Recibido el 16 de febrero de 2015; aceptado el 3 de noviembre de 2015

Disponible en Internet el 21 de febrero de 2017

\section{Resumen}

El objetivo del presente trabajo es analizar cómo se distribuye la capacidad tecnológica entre las entidades federativas de México y examinar su evolución. Para ello, se desarrolló un estudio empírico utilizando la técnica de análisis estadístico multivariante de cluster, con base en el set de indicadores propuesto por Cepal (2007) y recopilando los datos de diversas fuentes públicas del país para los años 2006 y 2012, con el fin de estudiar la evolución en el tiempo de dichos clusters, tratando de ver qué estados han podido mudarse a un cluster situado en posiciones más avanzadas y cuáles han retrocedido en dicho periodo. Los resultados muestran la existencia de 7 grupos de estados caracterizados por distintos niveles de capacidad tecnológica, y se detectan también 3 entidades que evolucionaron a un cluster más avanzado, tanto en lo referente a la capacidad de absorción e innovación, como en lo relativo a las capacidades tecnológicas de infraestructura. (C) 2017 Universidad Nacional Autónoma de México, Facultad de Contaduría y Administración. Este es un artículo Open Access bajo la licencia CC BY-NC-ND (http://creativecommons.org/licenses/by-nc-nd/4.0/).

Palabras clave: Capacidades tecnológicas; Clusters estatales; Análisis estadístico multivariante

Códigos JEL: O10; O30; O33

\footnotetext{
* Autor para correspondencia.

Correo electrónico: carolina.cph@ gmail.com (C.C. Pérez Hernández).

La revisión por pares es responsabilidad de la Universidad Nacional Autónoma de México.
} 


\begin{abstract}
This paper conducts an analysis on the existence of state clusters related with technological capabilities in Mexico. An empirical study was conducted using the technique of multivariate statistical cluster analysis, based on the set of indicators proposed by Cepal (2007), collecting data from various public sources country for 2006 and 2012 in order to study the time evolution of such clusters, trying to see what states have been moving to a cluster located in positions more which have advanced and retreated over the period. The results show the existence of 7 groups of states characterized by different technological capabilities, plus states are identified in decline and progress, both in terms of absorptive capacity and innovation, and in relation to the technological infrastructure capabilities.

(C) 2017 Universidad Nacional Autónoma de México, Facultad de Contaduría y Administración. This is an open access article under the CC BY-NC-ND license (http://creativecommons.org/licenses/by-nc-nd/4.0/).
\end{abstract}

Keywords: Technological capabilities; State clusters; Multivariate statistical analysis

JEL classification: $010 ; 030 ; 033$

\title{
Introducción
}

La ciencia, la tecnología y la innovación son los principales conductores del desarrollo económico sustentable (Stern, Porter y Furman, 2000; Schumpeter, 2005; Brunner, 2011; Dosi, 2008; Diaconu, 2011). Esto justifica la realización de estudios tanto nacionales como internacionales enfocados a medir las capacidades tecnológicas a nivel macro. Los estudios a nivel estatal no son tan numerosos. En México, el Foro Consultivo Científico y Tecnológico (FCCyT) se da a la tarea de presentar el estado de la Ciencia, Tecnología e Innovación (CTI) a nivel estatal-nacional. Sin embargo, tal como señala FCCyT (2014, p. 16), los estudios desarrollados en materia de medición de la CTI a nivel estatal «son incipientes y se requiere de análisis más amplios, complementarios o particulares».

Además, el concepto del sistema nacional de innovación es proclive a analizar las capacidades tecnológicas de diferentes entidades, ya que esto ayuda a entender de mejor manera sus transformaciones socioeconómicas (Dutrénit, Capdeville, Corona, Puchet y Vera-cruz, 2010). Se denota que la competitividad (internacional, nacional, estatal, industrial y empresarial) se construye. Es pues una ventaja adquirida y depende, esencialmente, de la amplitud y de la profundidad de las capacidades tecnológicas nacionales (Borrastero, 2012; Guzmán, 2008; Morales, 2009; Calderón y Hartmann, 2010; Close y Garita, 2011). En ese sentido, «las capacidades tecnológicas que impulsan la innovación han sido siempre un componente fundamental de la competitividad, el crecimiento y bienestar económico de los países» (Velarde, Garza y Coronado, 2011, p. 17).

El objetivo del presente trabajo es analizar cómo se distribuye la capacidad tecnológica entre las entidades federativas de México y examinar su evolución. Para ello, siguiendo a Blázquez y García (2009), se considera necesario realizar un análisis empírico que explore la existencia de diferentes grupos de estados (clusters) caracterizados por distintos niveles de capacidad tecnológica, de absorción y de innovación, evaluando la evolución de dichas capacidades entre los grupos de estados, para detectar aquellos que han logrado migrar a un cluster mejor posicionado y aquellos que han retrocedido en dicha materia. El estudio se ha realizado utilizando los datos de los años 2006 y 2012, publicados en diversas fuentes (tabla 1), los cuales contienen una serie de indicadores que tratan de cuantificar diferentes aspectos relacionados con la capacidad tecnológica. 
Tabla 1

Indicadores usados en el análisis empírico por estados para el año 2006 y 2012

Base disponible

La base disponible refleja los recursos humanos y la infraestructura, que revelan la «calidad» del entorno

\begin{tabular}{|c|c|c|}
\hline Variables & Definiciones & Fuente/año \\
\hline Líneas telefónicas & Densidad de líneas telefónicas fijas en servicio por entidad federativa. Líneas por cada cien habitantes & (CMIC, 2006, 2012) \\
\hline Usuarios de internet & Usuarios de internet y computadora por entidad federativa. Usuarios por cada 100 habitantes & (INEGI, 2012) \\
\hline Tasa de alfabetización & $\begin{array}{l}\text { Porcentaje de la población mayor a } 15 \text { años que sabe leer o escribir por entidad federativa. Población } \\
\text { alfabeta por cada } 100 \text { habitantes mayores de } 15 \text { años }\end{array}$ & (INEGI, 2006, 2012) \\
\hline Consumo de energía eléctrica & Consumo total de energía eléctrica per cápita por entidad federativa. Gigawatts por hora, per cápita & (CFE, 2006, 2012) \\
\hline $\begin{array}{l}\text { Investigadores dedicados a } \\
\text { investigación y desarrollo }\end{array}$ & $\begin{array}{l}\text { Investigadores pertenecientes al SNI dedicados a investigación y desarrollo por cada millón de habitantes } \\
\text { por entidad federativa }\end{array}$ & (CONACYT, 2006, 2012) \\
\hline $\begin{array}{l}\text { Titulados en Ingeniería y } \\
\text { Tecnología }\end{array}$ & Cantidad de personas tituladas en Ingeniería y Tecnología por entidad federativa & (ANUIES, 2006, 2012) \\
\hline Enrolamiento terciario & $\begin{array}{l}\text { Matrícula de estudiantes de educación superior, comprendida por licenciatura hasta cualquier posgrado. } \\
\text { Número de estudiantes por entidad federativa }\end{array}$ & (SEP, 2006, 2012) \\
\hline Enrolamiento secundario & $\begin{array}{l}\text { Matrícula de estudiantes de educación media superior, comprendida por profesional técnico y } \\
\text { bachillerato. Número de estudiantes por entidad federativa }\end{array}$ & $(\mathrm{SEP}, 2006,2012)$ \\
\hline Enrolamiento primario & $\begin{array}{l}\text { Matrícula de estudiantes de educación básica, comprendida por educación preescolar, primaria y } \\
\text { secundaria. Número de estudiantes por entidad federativa }\end{array}$ & (SEP, 2006, 2012) \\
\hline PIB & $\begin{array}{l}\text { Conjunto de bienes y servicios producidos en una entidad federativa durante un año. Millones de MX } \$ \text { a } \\
\text { precios constantes de } 2008\end{array}$ & (INEGI, 2006, 2012) \\
\hline PIB per cápita & Producto interno bruto per cápita por entidad federativa. MX\$ a precios constantes de 2008 & (INEGI, 2006, 2012) \\
\hline
\end{tabular}


Tabla 1

(continuación)

Los esfuerzos realizados

Esta dimensión refleja los esfuerzos realizados para el incremento y consolidación de las capacidades (adquisición de conocimiento en sus diversas formas, I+D, y otras)

\begin{tabular}{lll}
\hline Variables & Definiciones & Fuente/Año \\
\hline Gasto I+D empresas & Gasto destinado por las empresas del sector productivo en actividades de investigación y desarrollo & (INEGI, 2012)
\end{tabular}

Gasto I+D empresas $\quad$ Gasto destinado por las empresas del sector productivo en actividades de investigación y desarrollo (INEGI, 2012)

Inversión extranjera directa Inversión extranjera directa por entidad federativa. Millones de US\$ (SE, 2006, 2012)

Los resultados logrados

Esta dimensión muestra los resultados logrados a partir de las capacidades existentes (patentes, tasa de innovación y contenido tecnológico de las exportaciones)

\begin{tabular}{|c|c|c|}
\hline Variables & Definiciones & Fuente/Año \\
\hline $\begin{array}{l}\text { Artículos en publicaciones } \\
\text { científicas }\end{array}$ & Artículos publicados por titulares mexicanos en publicaciones científicas y técnicas por entidad federativa & (CONACYT, 2012) \\
\hline Patentes solicitadas & Patentes solicitadas en México por titulares mexicanos por entidad federativa por cada 100,000 habitantes & (IMPI, 2006, 2009) \\
\hline Patentes otorgadas & Patentes solicitadas en México por titulares mexicanos por entidad federativa por cada 100,000 habitantes & (IMPI, 2006, 2009) \\
\hline
\end{tabular}

Fuente: elaboración propia con base en la taxonomía de Cepal (2007). 
Con el trabajo empírico se analizan 2 periodos (2006 y 2012) a través de 3 etapas. La primera consiste en reducir un gran número de indicadores a través del análisis factorial, obteniéndose 2 factores. Después, tales factores se utilizan para identificar diferentes grupos de estados mediante la técnica de cluster. Finalmente, se realiza un test econométrico para evaluar la precisión estadística de los resultados de los conglomerados obtenidos, comprobando así la existencia de 7 grupos de estados caracterizados por distintos niveles de capacidad tecnológica y detectando también 3 entidades que evolucionaron a un cluster más avanzado, tanto en lo referente a la capacidad de absorción e innovación, como en lo relativo a las capacidades tecnológicas de infraestructura.

\section{Breve revisión de conceptos}

Bell, Pavitt y Lall (citados por Cepal, 2007, p. 11) señalan que el desarrollo de capacidades tecnológicas «implica conocimientos y habilidades para adquirir, usar, absorber, adaptar, mejorar y generar nuevas tecnologías». Partiendo de esta definición, se entiende que las capacidades tecnológicas incluyen las capacidades de innovación y las capacidades de absorción. La primera está sujeta a aspectos como las infraestructuras, las actividades de innovación y formación de capital humano, y las habilidades de los países para crear, imitar y gestionar el conocimiento, mientras que la segunda se refiere a la posibilidad de acceder, aprender y asimilar tecnologías extranjeras (Quiñones y Tezanos, 2011).

Para García, Blázquez y Ruiz (2012) existen 3 tipos de capacidades: la capacidad tecnológica, la capacidad de absorción y la capacidad de innovación (fig. 1), las cuales han sido usualmente tratadas por separado. Sin embargo, se ha evidenciado la existencia de elementos compartidos entre ellas y una intensa correlación que hace posible que dichas capacidades puedan ser estudiadas conjuntamente. Además, dado que las capacidades tecnológicas incluyen la capacidad de absorción e innovación, consecuentemente la conexión existente entre dichas capacidades hace pertinente la focalización hacia las capacidades tecnológicas como elemento central.

\section{Revisión de los indicadores utilizados}

Chinaprayoon (2007) indica que una de las peculiaridades de la tecnología es su variedad y, por lo tanto, las capacidades tecnológicas están compuestas de elementos heterogéneos, incluyendo las actividades de investigación, infraestructura, stock de conocimiento, recursos humanos y otros componentes. Debido a ello es imposible usar un solo indicador para explicar las capacidades tecnológicas de una nación o entidad.

En este artículo se utilizó una serie de indicadores que miden directa e indirectamente distintos aspectos relevantes de la capacidad tecnológica para las 32 entidades federativas de México. Es bien sabido que la ventaja de utilizar una batería de indicadores es que así se consigue definir con mayor precisión la situación de cada país (en este caso la situación estatal), proporcionando una comprensión más fácil de las diferencias entre ellos.

Propiamente, este trabajo adoptó las dimensiones propuestas por Cepal (2007), ya que dichas dimensiones toman en cuenta los sistemas de innovación para países en desarrollo (como México), los cuales merecen contar con su propia batería de indicadores basada en los componentes que son relevantes para su contexto. En ese sentido, se adaptaron algunas variables en relación a la dimensión de los esfuerzos realizados y los resultados logrados, ya que, como indican Archibugi y Coco (2005), en muchos casos las variables son dictadas por la disponibilidad de las fuentes estadísticas, más que de las preferencias teóricas. Para este estudio se tomaron los años 2006 y 2012, por tratarse de información pública disponible (tabla 1). 


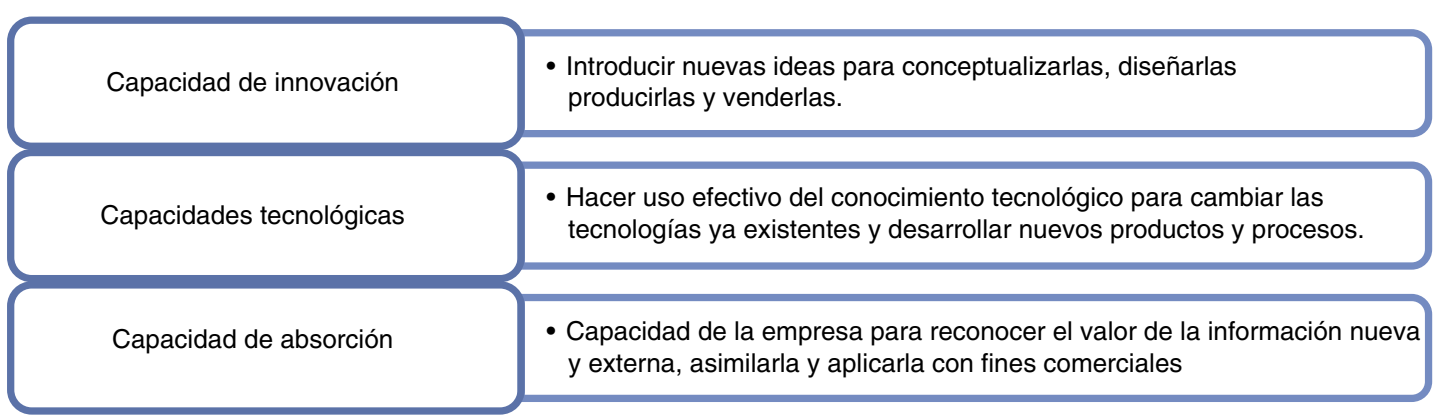

Figura 1. Tipos de capacidades.

Fuente: elaboración propia con base en Biggs, Shah y Srivastava (1995) y García et al. (2012a). 
Es importante indicar que los datos que se han utilizado para este trabajo provienen de 2 fuentes: datos publicados referentes al año 2006 y 2012 (datos duros) y datos de la Encuesta sobre Investigación y Desarrollo Tecnológico y Módulo sobre Actividades de Biotecnología y Nanotecnología (datos de encuesta) ${ }^{1}$.

Para lograr que los datos sean comparables se ha procedido a normalizarlos de acuerdo con la fórmula para comparar indicadores individuales propuesta por Archibugi y Coco (2004), la cual se expresa como sigue: (valor observado - valor mínimo) / (valor máximo - valor mínimo); rango de índices: [0 y 1].

\section{Metodología y análisis de resultados}

La metodología del presente trabajo consiste en desarrollar un análisis estadístico multivariante de cluster para los años 2006 y 2012 que, como primer punto, muestre los resultados referentes a la estadística descriptiva de las variables previamente normalizadas; posteriormente se realiza el análisis factorial, para destacar los principales componentes relativos al estudio de la capacidad tecnológica, y como tercer paso se corre un análisis de conglomerados jerárquicos, mejor conocido como análisis cluster, para identificar los grupos de estados que comparten características similares. Finalmente, se realiza un test econométrico para la validación del análisis cluster. Los métodos mencionados se describirán puntualmente en las siguientes secciones.

\section{Revisión de los estadísticos descriptivos}

En esta sección se presentan los estadísticos descriptivos previamente normalizados para los años analizados, en los cuales se considera que al tomar el dato menor y aplicarle la fórmula de normalización, se obtiene un cero. Asimismo, el dato mayor será un uno. Por lo tanto, estos datos definen el rango $[0,1]$ y todos los demás datos estarán contenidos en dicho intervalo. Podría entenderse que el dato se vuelve un porcentaje, donde el $0 \%$ es el valor mínimo y el $100 \%$ es el valor máximo, y las variables en cuestión representan algún porcentaje entre 0 y 100.

Los estadísticos descriptivos que resultan útiles en el análisis son la media y la desviación típica (tal como se muestra en la tabla 2). Para la mayoría de las variables la media tiene un crecimiento pequeño y la desviación típica se mantiene igual o casi igual, lo que implica que la mayoría de los estados crecieron aproximadamente en la misma cantidad.

Sin embargo, variables como la inversión extranjera directa o el PIB per cápita muestran un crecimiento importante en la media. No obstante, la desviación típica se mantiene muy parecida. Esto indicaría que la mayoría de los estados aumentaron su valor en estas variables de forma importante pero mantuvieron la misma dispersión en sus diferencias con respecto a los demás estados. Podría interpretarse que hubo un cierto factor que afectó a todos los estados causando el aumento generalizado en los valores.

Por otro lado, variables como las patentes otorgadas, el número de investigadores y el PIB aumentaron en gran medida en sus medias y también sufrieron cambios importantes en sus desviaciones típicas. Esto quiere decir que los estados crecieron pero de forma dispareja, unos más que otros. Puede interpretarse que para estas variables no hubo un solo factor que produjera los

\footnotetext{
${ }^{1}$ La encuesta se conoce como «datos blandos», y por lo general se obtiene de muestras de las encuestas (no de poblaciones enteras). Para el INEGI, la ESIDET-MBN 2012 es la primera encuesta especial en empresas con cobertura geográfica estatal; por lo tanto, la batería del año 2006 no emplea dicha variable.
} 
Tabla 2

Estadísticos descriptivos (año 2006 y 2012)

\begin{tabular}{|c|c|c|c|c|c|c|}
\hline \multirow[t]{2}{*}{ Variables } & \multirow{2}{*}{$\begin{array}{l}\text { Media } \\
2006\end{array}$} & \multirow{2}{*}{$\begin{array}{l}\text { Media } \\
2012\end{array}$} & \multirow[t]{2}{*}{ Variación \% } & \multirow{2}{*}{$\begin{array}{l}\text { Desv. típ. } \\
2006\end{array}$} & \multirow{2}{*}{$\begin{array}{l}\text { Desv. típ. } \\
2012\end{array}$} & \multirow[t]{2}{*}{ Variación \% } \\
\hline & & & & & & \\
\hline Inversión extranjera directa & 0.066 & 0.088 & 33 & 0.175 & 0.175 & 0 \\
\hline Patentes otorgadas & 0.067 & 0.124 & 85 & 0.178 & 0.240 & 35 \\
\hline Investigadores & 0.068 & 0.169 & 149 & 0.173 & 0.199 & 15 \\
\hline PIB per cápita & 0.088 & 0.114 & 30 & 0.172 & 0.174 & 1 \\
\hline Patentes solicitadas & 0.098 & 0.167 & 70 & 0.198 & 0.222 & 12 \\
\hline PIB millones de $\mathrm{MX} \$$ & 0.152 & 0.155 & 2 & 0.196 & 0.196 & 0 \\
\hline Enrolamiento terciario bruto & 0.162 & 0.171 & 6 & 0.2 & 0.209 & 5 \\
\hline Enrolamiento primario bruto & 0.211 & 0.206 & -2 & 0.206 & 0.203 & -1 \\
\hline Enrolamiento secundario bruto & 0.228 & 0.218 & -4 & 0.237 & 0.222 & -6 \\
\hline Líneas telefónicas & 0.322 & 0.248 & -23 & 0.192 & 0.184 & -4 \\
\hline Consumo de energía & 0.419 & 0.419 & 0 & 0.256 & 0.256 & 0 \\
\hline Tasa de alfabetización & 0.697 & 0.703 & 1 & 0.267 & 0.268 & 0 \\
\hline Publicaciones científicas & N.A. & 0.062 & N.A. & N.A. & 0.174 & N.A. \\
\hline Usuarios Internet & N.A. & 0.484 & N.A. & N.A. & 0.282 & N.A. \\
\hline Gasto I+D (empresas) & N.A. & 0.06 & N.A. & N.A. & 0.178 & N.A. \\
\hline Titulados en Ingeniería y Tecnología & N.A. & 0.173 & N.A. & N.A. & 0.199 & N.A. \\
\hline
\end{tabular}

N.A.: dato no disponible en dicho año. $\mathrm{n}$ válido $=32$ (según lista).

Fuente: elaboración propia (SPSS 21).

cambios en todos los estados, sino que cada uno cambió por sus propias causas. Finalmente, cabe recalcar que la variable líneas telefónicas sufrió un decremento en su media; dicha variable, según Chinaprayoon (2007), es considerada como difusora de las viejas tecnologías, en contraste con internet y las líneas de teléfono celular, que representan la difusión de las nuevas tecnologías.

\section{Análisis factorial}

El análisis factorial tiene como objetivo identificar las variables explicativas que mejor examinan la distribución de la capacidad tecnológica entre estados. El objetivo del análisis factorial es, por tanto, extraer un número menor de factores que expliquen la mayor parte de la varianza de la muestra, y es una técnica ampliamente utilizada y aceptada en este tipo de estudios (Archibugi, 1988). Sin embargo, previo al análisis factorial se estudió la viabilidad de realizarlo para el conjunto de datos (años 2006 y 2012), para lo cual se utilizó la prueba de Kaiser-Meyer-Okin (KMO) y la prueba de esfericidad de Bartlett (tabla 3).

Tabla 3

KMO y prueba de Bartlett año 2006 y 2012

\begin{tabular}{lll}
\hline KMO y prueba de Bartlett & Año 2006 & Año 2012 \\
\hline Medida de adecuación muestral de Kaiser-Meyer-Olkin & 0.763 & 0.717 \\
Prueba de esfericidad de Bartlett & & 865.127 \\
Chi-cuadrado aproximado & 662.559 & 120 \\
gl & 66 & 0.000 \\
Sig. & 0.000 & \\
\hline
\end{tabular}

Fuente: elaboración propia (SPSS 21). 
Tabla 4

Resultados de la matriz de componentes rotados

Factor 1

Factor 2

Capacidad de absorción e innovación

Capacidad tecnológica de infraestructura

Graficado en:

Eje de las Y

Eje de las X

Enrolamiento terciario

Titulados (Ingeniería \& Tecnología)

PIB precios constantes

Gasto I+D (empresas)

Inversión extranjera directa

Usuarios de internet

Tasa de alfabetización

Consumo de eléctrica

Publicaciones científicas

PIB per cápita

Enrolamiento secundario

Enrolamiento primario

Patentes solicitadas

Investigadores

Patentes otorgadas

Para cada factor se eligen las variables con saturaciones superiores a 0.5 .

Fuente: elaboración propia con base en resultados Matriz de Componentes rotados (SPSS 21).

El índice de KMO se utiliza para comparar las magnitudes de los coeficientes de correlación múltiples observados con las magnitudes de coeficientes de correlación parcial (Álvarez, 1995). Cuando el valor del índice es bajo, menor de 0.5, se desaconseja la aplicación del análisis, ya que las correlaciones entre pares de variables no se pueden explicar a través de las otras variables. Cuanto más próximo a 1 esté el índice KMO, más adecuada es la utilización del análisis factorial. Se observa que para el año 2006 el índice $\mathrm{KMO}=0.763>0.5$. Entonces sí tiene sentido hacer un análisis factorial. Lo mismo sucede para el año 2012, ya que el índice KMO =0.717>0.5.

Por otra parte, la prueba de esfericidad de Bartlett contrasta si hay interrelaciones entre las variables mediante la enunciación de la hipótesis nula, consistente en que la matriz de correlación es la matriz identidad (la que tiene unos en la diagonal principal y ceros en el resto de valores). Si se confirma la hipótesis nula, supondría que las variables no están correlacionadas. Si por el contrario se rechaza la hipótesis nula, las variables estarían relacionadas y sería adecuado realizar el análisis factorial (Pedroza, 2006).

Para ambos periodos 2006 y 2012, el valor p asociado a la prueba de esfericidad de Bartlett es menor a 0.05 , entonces se rechaza la $\mathrm{H}_{0} \mathrm{y}$, por lo tanto, sí tiene sentido hacer un análisis factorial. La tabla 4 agrupa los resultados de los factores en los años 2006 y 2012, respectivamente. Estos 2 factores conjuntamente explican un alto porcentaje de la varianza de la muestra (entre 74.26 y $78.65 \%$, respectivamente), lo cual es significativo a niveles convencionales e indica, por tanto, que estos factores representan la mayor parte de la variabilidad en los datos.

Factor 1. Es una combinación de 11 variables que suponen el 58.5\% de la varianza de la muestra en el año 2006 y el $54.7 \%$ en el año 2012, por lo que implica ser una dimensión muy relevante para analizar las diferencias en la capacidad tecnológica entre entidades federativas. El factor 1, acorde con la literatura, podría ser interpretado como la capacidad de absorción e innovación.

Factor 2. Es una combinación de 5 variables que suponen el $19.5 \%$ de la varianza de la muestra (año 2012) y el $20.1 \%$ en el año 2006. El factor 2, acorde con la literatura, podría ser interpretado como la capacidad tecnológica de infraestructura. 
La tabla 4, denominada «matriz de componentes rotados», indica la correlación existente (saturación) entre cada una de las variables y su correspondiente factor. La saturación representa el peso (la importancia) de la variable dentro del factor. Cabe mencionar que a diferencia de lo que ocurre en otras técnicas como el análisis de varianza o el de regresión, en el análisis factorial todas las variables del análisis cumplen el mismo papel: todas ellas son independientes en el sentido de que no existe a priori una dependencia conceptual de unas variables sobre otras. Por lo tanto, las variables de ambos factores (X \& Y) son consideradas independientes.

Habrá que poner en claro el significado de los factores obtenidos. El factor 1 (graficado en el eje de las Y), denominado capacidad de absorción e innovación, incluye variables relativas a la tasa de enrolamiento, recursos humanos dedicados a la investigación, ciencia y tecnología; dichas variables suponen la capacidad de los estados para reconocer el valor de la información nueva y externa, asimilarla y aplicarla con fines comerciales. Por otra parte, dentro de este mismo factor se incluyen variables de resultados logrados, como lo son las patentes solicitadas y otorgadas y el gasto en investigación y desarrollo de las empresas por estado, indicadores que muestran la capacidad de las entidades federativas para introducir nuevas ideas, conceptualizarlas, diseñarlas, producirlas y venderlas. Otra de las variables incluida en el factor 1 es la referente a la inversión extranjera directa, que ejemplifica la adquisición del conocimiento externo de las entidades.

Por otra parte, el factor 2, etiquetado como capacidad tecnológica de infraestructura, considera indicadores de consumo de energía eléctrica, líneas de teléfono y usuarios de internet ${ }^{2}$, variables consideradas como de infraestructura que aportan un conocimiento general del entorno en el cual se desarrollan las actividades productivas de las entidades de la república mexicana. La combinación de estos 3 aspectos ofrece indicios del grado de sofisticación de la producción, «ya que puede suponerse que a mayor valor de los indicadores en cuestión corresponde una mayor sofisticación, lo que debería traducirse en mayor valor agregado en la producción» (Cepal, 2007, p. 32). Además, dicho factor añade la tasa de alfabetización como una métrica del nivel general del entorno productivo. Finalmente, se incluye el indicador del PIB per cápita, ya que es sabido que los productos con mayor contenido tecnológico (o contenido de conocimiento) se caracterizan por una mayor elasticidad de la demanda. En otros el PIB per cápita es un indicador de la complejidad de la demanda tecnológica ${ }^{3}$.

\section{Análisis cluster}

El análisis cluster es la denominación de un grupo de técnicas multivariantes cuyo principal propósito es agrupar objetos basándose en las características que poseen. Los conglomerados resultantes deberían mostrar un alto grado de homogeneidad interna dentro del conglomerado y un alto grado de heterogeneidad externa del mismo (Álvarez, 1995). En este caso se busca la partición de un conjunto de datos (correspondientes a distintos estados) en grupos, de tal forma que los datos pertenecientes a un mismo grupo sean muy similares entre sí pero muy diferentes a los de los otros grupos. Para conseguir formar grupos homogéneos de observaciones (en este caso de entidades) hay que medir su similaridad o su distancia (disimilaridad). A este respecto, se han desarrollado numerosos métodos para medir la distancia entre los casos. En este trabajo

\footnotetext{
2 Para Chinaprayoon (2007), los usuarios de internet son un indicador de la difusión de nuevas tecnologías, mientras que el consumo de energía eléctrica y las líneas de teléfono indican la difusión de las viejas tecnologías.

${ }^{3}$ Se espera que el crecimiento de la actividad económica y el ingreso derive en un aumento de la demanda de bienes de mayor complejidad o tecnológicamente avanzados (Cepal, 2007).
} 
se utilizó la distancia euclídea, la cual mide el parecido entre unidades de análisis que han sido evaluadas en un conjunto de variables métricas (cuantitativas).

\section{Los clusters estatales y su evolución en el período 2006-2012}

El análisis de conglomerados jerárquicos (análisis cluster) comienza con el cálculo de la matriz de distancias entre los elementos de la muestra. Esa matriz contiene las distancias existentes entre cada elemento y todos los restantes de la muestra. Posteriormente se buscan los 2 elementos más próximos (es decir, los 2 más similares en términos de distancia) y se agrupan en un conglomerado. El conglomerado resultante es indivisible a partir de ese momento (de ahí el nombre de jerárquico asignado al procedimiento). De esta manera, se van agrupando los elementos en conglomerados cada vez más grandes y más heterogéneos entre ellos, hasta llegar al último paso, en el que todos los elementos muestrales quedan agrupados en un único conglomerado global. El procedimiento de conglomerados jerárquico del SPSS informa de todos los pasos realizados en el análisis, por lo que resulta fácil apreciar qué elementos o conglomerados se han fundido en cada paso y a qué distancia se encontraban cuando se fusionaron. Esto permite valorar la heterogeneidad de los conglomerados. Esta sección presenta los resultados del análisis estadístico multivalente cluster que divide las entidades federativas de México en 7 grupos o clusters caracterizados por distintos niveles de capacidad tecnológica en los períodos 2006 y 2012. En la tabla 5 aparecen los estados que integran cada uno de los grupos en los 2 períodos considerados.

En materia de capacidades tecnológicas, México se mueve a 7 pasos diferentes. Los clusters estatales de capacidad tecnológica obtenidos son descritos a continuación:

Clúster 1. Excelente en capacidad de absorción e innovación y bueno en capacidad tecnológica de infraestructura. En este conglomerado se encuentra únicamente el D.F., en los 2 periodos de tiempo, al contar con una posición claramente destacada en cuanto a estos 2 componentes. Sin embargo, no se ha producido una evolución visible en ninguno de los 2 factores en los períodos considerado. De modo contrario, parece haber disminuido ligeramente en los factores analizados de 2006 y 2012.

Clúster 2. Regular en capacidad de absorción e innovación y excelente en capacidad tecnológica de infraestructura. Este grupo se encuentra a una distancia considerable respecto del cluster 1, formado por D.F., en cuanto a capacidad de absorción e innovación. Sin embargo, es el grupo que obtiene una mejor posición en cuanto a capacidad tecnológica de infraestructura. Este conglomerado estaba formado en 2006 únicamente por Nuevo León; sin embargo, en 2012 este grupo está compuesto por Nuevo León y Querétaro, este último estado proveniente del cluster 6 en el año 2006, debido fundamentalmente a su mejora en ambos factores.

Clúster 3. Bueno en capacidad de absorción e innovación y con déficit bajo en capacidad tecnológica de infraestructura. En este conglomerado se encuentra Jalisco, tanto en 2006 como en 2012. Por una parte, se encuentra bien posicionado dentro del componente de capacidad de absorción e innovación, pero con un posicionamiento ligeramente negativo en lo relativo a las capacidades tecnológicas de infraestructura.

Clúster 4. Bueno en capacidad de absorción e innovación y con déficit medio en capacidad tecnológica de infraestructura. En este conglomerado se encuentra el estado de México; se observa que en el primer componente presenta una posición positiva (solo por debajo del cluster 1), y en lo que respecta al segundo componente, se ubica en el cuadrante negativo.

Clúster 5. Déficit bajo en capacidad de absorción e innovación y regular en capacidad tecnológica de infraestructura. Este grupo estaba constituido por 9 estados en 2006 (Aguascalientes, Baja 
Tabla 5

Clusters obtenidos

\begin{tabular}{|c|c|c|}
\hline Cluster & 2006 & 2012 \\
\hline 1 & D.F. & D.F. \\
\hline 2 & Nuevo León & $\begin{array}{l}\text { Nuevo León } \\
\text { Querétaro* }\end{array}$ \\
\hline 3 & Jalisco & Jalisco \\
\hline 4 & México & México \\
\hline \multirow[t]{11}{*}{5} & Aguascalientes & Aguascalientes \\
\hline & Baja California Norte & Baja California Norte \\
\hline & Baja California Sur & Baja California Sur \\
\hline & Coahuila & Campeche* \\
\hline & Colima & Coahuila \\
\hline & Chihuahua & Colima \\
\hline & Quintana Roo & Chihuahua \\
\hline & Sonora & Morelos* \\
\hline & Tamaulipas & Quintana Roo \\
\hline & & Sonora \\
\hline & & Tamaulipas \\
\hline \multirow[t]{14}{*}{6} & Campeche* & Durango \\
\hline & Durango & Guanajuato \\
\hline & Guanajuato & Hidalgo \\
\hline & Hidalgo & Michoacán \\
\hline & Michoacán & Nayarit \\
\hline & Morelos* & San Luis Potosí \\
\hline & Nayarit & Sinaloa \\
\hline & Querétaro* & Tabasco \\
\hline & San Luis Potosí & Tlaxcala \\
\hline & Sinaloa & Yucatán \\
\hline & Tabasco & Zacatecas \\
\hline & Tlaxcala & \\
\hline & Yucatán & \\
\hline & Zacatecas & \\
\hline \multirow[t]{5}{*}{7} & Veracruz & Veracruz \\
\hline & Puebla & Puebla \\
\hline & Guerrero & Guerrero \\
\hline & Oaxaca & Oaxaca \\
\hline & Chiapas & Chiapas \\
\hline
\end{tabular}

Fuente: elaboración propia con base en resultados de conglomerados (SPSS 21).

* Los estados marcados con asterisco (Querétaro, Morelos, Campeche) son aquellos que lograron migrar a un cluster más avanzado.

California Norte, Baja California Sur, Coahuila, Colima, Chihuahua, Quintana Roo, Sonora, Tamaulipas). En 2012, todos esos estados permanecen en este grupo, no observándose ninguna evolución. Sin embargo, para esa fecha se les añaden los estados de Campeche y Morelos (los cuales se encontraban en el cluster 6 en el año 2006) y cuyo progreso se debió principalmente a la mejoría en ambos factores.

Clúster 6. Déficit medio en capacidad de absorción e innovación y déficit medio en capacidad tecnológica de infraestructura. Este grupo estaba constituido por 14 estados en 2006 (Campeche, Durango, Guanajuato, Hidalgo, Michoacán, Morelos, Nayarit, Querétaro, San Luis Potosí, Sinaloa, Tabasco, Tlaxcala, Yucatán y Zacatecas). En 2012, todos esos estados permanecen en este grupo, con excepción de Campeche, Morelos y Querétaro, los cuales migraron a clusters en 
Tabla 6

Prueba ANOVA de 2 factores. Año 2006

\begin{tabular}{|c|c|c|c|c|c|}
\hline \multicolumn{6}{|c|}{ ANOVA de 2 factores } \\
\hline & Suma de cuadrados & $\mathrm{gl}$ & Media cuadrática & $\mathrm{F}$ & Sig. \\
\hline \multicolumn{6}{|c|}{ REGR factor score 1 for analysis 1} \\
\hline Inter-grupos & 29.547 & 6 & 4.924 & 84.700 & 0.000 \\
\hline Intra-grupos & 1.453 & 25 & 0.058 & & \\
\hline Total & 31.000 & 31 & & & \\
\hline \multicolumn{6}{|c|}{ REGR factor score 2 for analysis 1} \\
\hline Inter-grupos & 28.086 & 6 & 4.681 & 40.153 & 0.000 \\
\hline Intra-grupos & 2.914 & 25 & 0.117 & & \\
\hline Total & 31.000 & 31 & & & \\
\hline
\end{tabular}

Fuente: elaboración propia (SPSS 21).

posiciones más avanzadas. Campeche y Morelos avanzaron al cluster 5, mientras que el estado de Querétaro proviene del cluster 6 (2006) al cluster 2 (2012), debido a una ligera mejora en el factor 1 y una muy amplia mejora en lo referente al factor 2 .

Clúster 7. Déficit bajo en capacidad de absorción e innovación y déficit elevado en capacidad tecnológica de infraestructura: Este grupo estaba constituido por 5 estados en 2006 (Veracruz, Puebla, Guerrero, Oaxaca, Chiapas). En 2012, todos esos estados permanecen en este grupo. Se observa que los estados de Veracruz y Puebla se hallan en el límite del grupo, tendentes a migrar a un cluster más avanzado.Estos clusters así definidos se pueden observar con más claridad en la figura 2, que muestran a todos los estados ordenados en función de los 2 factores obtenidos.

\section{Test econométrico de validación del análisis cluster para el año 2006}

En el mismo sentido, se ha llevado a cabo un test ANOVA (tabla 6) para contrastar que el análisis cluster realizado para el año 2006 es adecuado y si existen diferencias significativas entre los grupos obtenidos. El ANOVA y las pruebas post-hoc nos permitirán verificar que el análisis de clusters efectuado para las distintas variables es correcto, en el sentido de poder comprobar la existencia de diferencias significativas entre los 7 grupos considerados. Los resultados que se muestran a continuación confirman la bondad del análisis.

Dentro del test ANOVA (año 2006), dado que $\mathrm{p}=0.05>\mathrm{Sig}=0.000$, se rechaza la $\mathrm{H}_{0}$, y por lo tanto existe una diferencia estadísticamente significativa entre el total de grupos.

Una vez que se ha determinado que existen diferencias entre las medias, las pruebas de rango post-hoc permiten determinar qué medias difieren. La prueba de rango post-hoc identifica subconjuntos homogéneos de medias que no se diferencian entre sí. Por tanto, para comprobar si existen diferencias entre todos los grupos se han realizado además las pruebas de Student-Newman-Keuls, HDS de Tukey y Waller-Duncan ${ }^{4}$. Se ha efectuado la prueba con los 3 grupos que contienen más

\footnotetext{
${ }^{4}$ El test Student-Newman-Keuls es un test de comparaciones múltiples, permite comparar las medias de los niveles $\mathrm{t}$ de un factor después de haber rechazado la $\mathrm{H}_{0}$ de igualdad de medias mediante la técnica ANOVA. La prueba DHS de Tukey utiliza el estadístico del rango estudentizado para realizar todas las comparaciones por pares entre los grupos y establece la tasa de error por experimento como la tasa de error para el conjunto de todas las comparaciones por pares. La prueba Waller-Duncan utiliza la aproximación bayesiana. Esta prueba de rango emplea la media armónica del tamaño de la muestra cuando los tamaños muestrales no son iguales (Martín, Cabero y de Paz, 2008).
} 

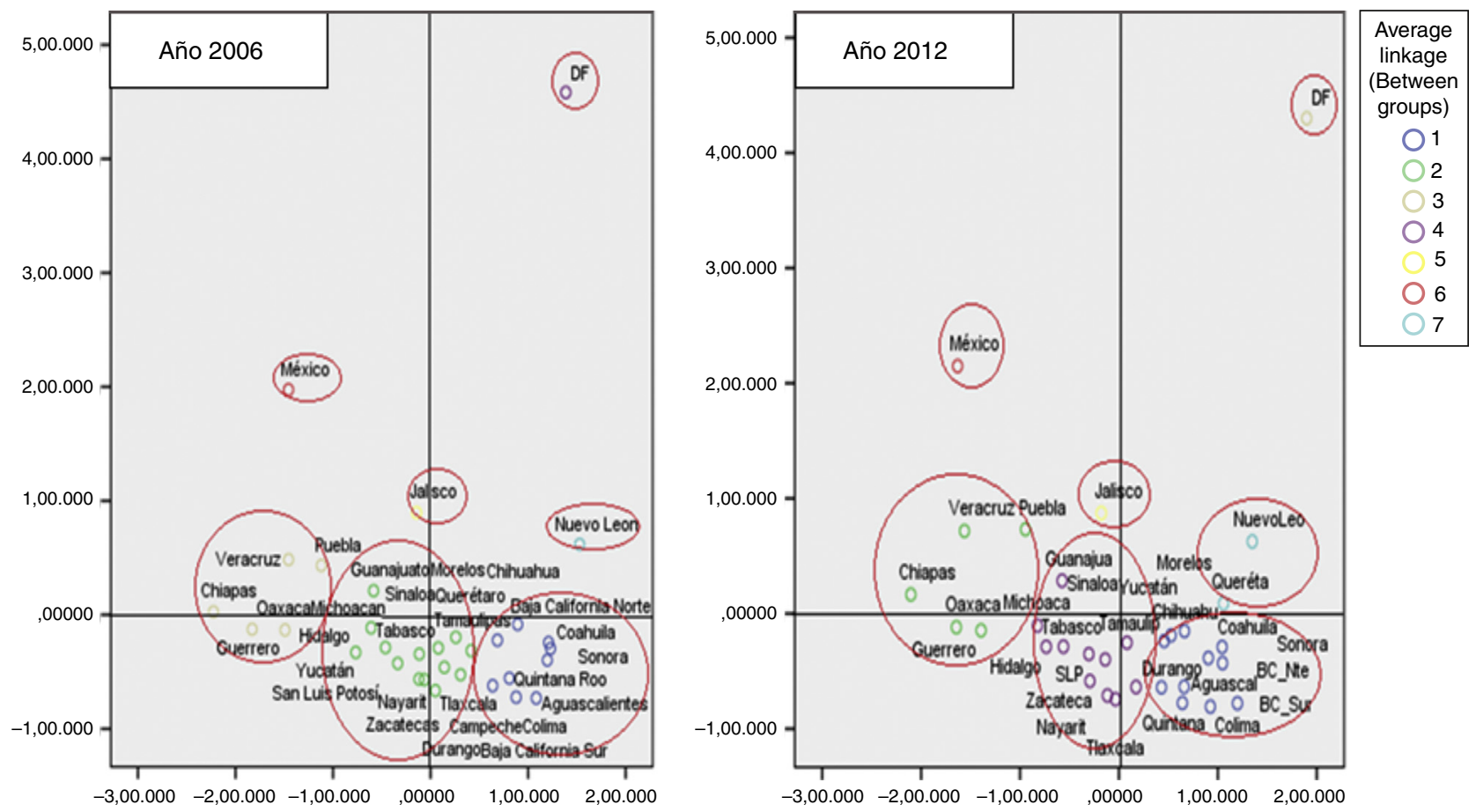

Eje Y: Capacidad de absorción e innovación

Eje X: Capacidad tecnológica de infraestructura

Figura 2. Gráficos de dispersión 2006 y 2012.

Fuente: elaboración propia (SPSS 21). 
Tabla 7

Pruebas post-hoc para el factor 1-2. Año 2006

\begin{tabular}{|c|c|c|c|c|c|c|c|c|c|c|}
\hline \multirow{2}{*}{$\begin{array}{l}\text { Factor } 1 \text { (año } \\
2006 \text { ) }\end{array}$} & \multirow{2}{*}{$\begin{array}{l}\text { Average Linkage } \\
\text { (Between Groups) }\end{array}$} & \multirow[t]{2}{*}{$\mathrm{n}$} & \multicolumn{2}{|c|}{ Subconjunto } & \multirow{2}{*}{$\begin{array}{l}\text { Factor } 2 \text { (año } \\
2006)\end{array}$} & \multirow{2}{*}{$\begin{array}{l}\text { Average Linkage } \\
\text { (Between Groups) }\end{array}$} & \multirow[t]{2}{*}{$\mathrm{n}$} & \multicolumn{3}{|c|}{ Subconjunto } \\
\hline & & & 1 & 2 & & & & 1 & 2 & 3 \\
\hline Student-Newman- & Cluster 5 & 9 & -0.4306927 & & Student-Newman- & Cluster 7 & 5 & -1.6247981 & & \\
\hline \multirow[t]{3}{*}{ Keuls $^{\mathrm{a}, \mathrm{b}}$} & Cluster 6 & 14 & -0.3483875 & & Keuls $^{\mathrm{a}, \mathrm{b}}$ & Cluster 6 & 14 & & -0.1291626 & \\
\hline & Cluster 7 & 5 & & 0.1364949 & & Cluster 5 & 9 & & & 0.9575606 \\
\hline & Sig. & & 0.505 & 1 & & Sig. & & 1 & 1 & 1 \\
\hline \multirow[t]{4}{*}{ DHS de Tukey ${ }^{\mathrm{a}, \mathrm{b}}$} & Cluster 5 & 9 & -0.4306927 & & DHS de Tukey ${ }^{\mathrm{a}, \mathrm{b}}$ & Cluster 7 & 5 & -1.6247981 & & \\
\hline & Cluster 6 & 14 & -0.3483875 & & & Cluster 6 & 14 & & -0.1291626 & \\
\hline & Cluster 7 & 5 & & 0.1364949 & & Cluster 5 & 9 & & & 0.9575606 \\
\hline & Sig. & & 0.779 & 1 & & Sig. & & 1 & 1 & 1 \\
\hline \multirow[t]{3}{*}{ Waller-Duncan ${ }^{\mathrm{a}, \mathrm{c}}$} & Cluster 5 & 9 & -0.4306927 & & Waller-Duncan ${ }^{\mathrm{a}, \mathrm{c}}$ & Cluster 7 & 5 & -1.6247981 & & \\
\hline & Cluster 6 & 14 & -0.3483875 & & & Cluster 6 & 14 & & -0.1291626 & \\
\hline & Cluster 7 & 5 & & 0.1364949 & & Cluster 5 & 9 & & & 0.9575606 \\
\hline \multicolumn{5}{|c|}{$\begin{array}{l}\text { Se muestran las medias de los grupos de subconjuntos } \\
\text { homogéneos. Basadas en las medias observadas. El término } \\
\text { de error es la media cuadrática (Error) }=0.058 \text {. }\end{array}$} & \multicolumn{6}{|c|}{$\begin{array}{l}\text { Se muestran las medias de los grupos de subconjuntos } \\
\text { homogéneos. Basadas en las medias observadas. El término } \\
\text { de error es la media cuadrática (Error) }=0.117 \text {. }\end{array}$} \\
\hline
\end{tabular}


Tabla 8

Prueba ANOVA de 2 factores. Año 2012

\begin{tabular}{|c|c|c|c|c|c|}
\hline \multicolumn{6}{|c|}{ ANOVA de un factor } \\
\hline & Suma de cuadrados & $\mathrm{gl}$ & Media cuadrática & $\mathrm{F}$ & Sig. \\
\hline \multicolumn{6}{|c|}{ REGR factor score 1 for analysis 5 (factor 1 ) } \\
\hline Inter-grupos & 28.870 & 6 & 4.812 & 56.465 & 0.000 \\
\hline Intra-grupos & 2.130 & 25 & 0.085 & & \\
\hline Total & 31.000 & 31 & & & \\
\hline \multicolumn{6}{|c|}{ REGR factor score 2 for analysis 5 (factor 2 ) } \\
\hline Inter-grupos & 28.440 & 6 & 4.740 & 46.287 & 0.000 \\
\hline Intra-grupos & 2.560 & 25 & 0.102 & & \\
\hline Total & 31.000 & 31 & & & \\
\hline
\end{tabular}

Fuente: elaboración propia (SPSS 21).

de un estado, eliminando por tanto el caso del D.F., México, Jalisco y Nuevo León, que presenta claras diferencias con el resto de grupos.

La tabla 7 contiene los resultados de las pruebas post-hoc para el factor 1 definido como las capacidades de absorción e innovación. Se observa que:

- Existe una diferencia estadísticamente significativa entre el cluster 5 y el cluster 7.

- Existe una diferencia estadísticamente significativa entre el cluster 6 y el cluster 7.

- Sin embargo, no se presentan diferencias significativas entre el cluster 5 y el cluster 6 , debido a que la Sig. $>0.05$, en este caso 0.5>0.05. En el diagrama de dispersión (fig. 2) se puede observar cierta coincidencia.

Del mismo modo, los resultados de las pruebas post-hoc para el factor 2, definido como las capacidades tecnológicas de infraestructura, muestran que existe una diferencia estadísticamente significativa entre todos los clusters.

\section{Test econométrico de validación del análisis cluster para el año 2012}

De igual manera, se ha llevado a cabo el test ANOVA para contrastar que el análisis cluster realizado para el año 2012 es adecuado y si existen diferencias significativas entre los grupos obtenidos (tabla 8).

Los resultados de la prueba ANOVA rechazan la $\mathrm{H}_{0}$. Por lo tanto, existe una diferencia estadísticamente significativa entre el total de grupos, dado que $\mathrm{p}=0.05>\mathrm{Sig}=0.000$.

Se han efectuado también las pruebas post-hoc con los 4 grupos que contienen más de un estado, eliminando por tanto el caso del D.F., México y Jalisco, que presenta claras diferencias con el resto de los grupos.

La tabla 9 muestra los resultados de las pruebas post-hoc para el factor 1, definido como las capacidades de absorción e innovación, en donde se observa que:

- Existe una diferencia estadísticamente significativa entre el cluster 5 y el cluster 7.

- Existe una diferencia estadísticamente significativa entre el cluster 5 y el cluster 2.

- Existe una diferencia estadísticamente significativa entre el cluster 6 y el cluster 7.

- Existe una diferencia estadísticamente significativa entre el cluster 6 y el cluster 2. 
Tabla 9

Pruebas post-hoc para el factor 1-2. Año 2012

\begin{tabular}{|c|c|c|c|c|c|c|c|c|c|c|}
\hline \multirow{2}{*}{$\begin{array}{l}\text { Factor } 1 \text { (año } \\
\text { 2012) }\end{array}$} & \multirow{2}{*}{$\begin{array}{l}\text { Average Linkage } \\
\text { (Between Groups) }\end{array}$} & \multirow[t]{2}{*}{$\mathrm{n}$} & \multicolumn{2}{|c|}{ Subconjunto } & \multirow{2}{*}{$\begin{array}{l}\text { Factor } 2 \text { (año } \\
2012 \text { ) }\end{array}$} & \multirow{2}{*}{$\begin{array}{l}\text { Average Linkage } \\
\text { (Between Groups) }\end{array}$} & \multirow[t]{2}{*}{$\mathrm{n}$} & \multicolumn{3}{|c|}{ Subconjunto } \\
\hline & & & 1 & 2 & & & & 1 & 2 & 3 \\
\hline Student- & Cluster 5 & 11 & -0.4538141 & & Student- & Cluster 7 & 5 & -1.5369134 & & \\
\hline Newman- & Cluster 6 & 11 & -0.3682284 & & Newman-Keuls ${ }^{\mathrm{a}, \mathrm{c}}$ & Cluster 6 & 11 & & -0.2790508 & \\
\hline \multirow[t]{3}{*}{ Keuls $^{\mathrm{a}, \mathrm{b}, \mathrm{c}}$} & Cluster 7 & 5 & & 0.208895 & & Cluster 5 & 11 & & & 0.7911984 \\
\hline & Cluster 2 & 2 & & 0.3759092 & & Cluster 2 & 2 & & & 1.1697297 \\
\hline & Sig. & & 0.663 & 0.397 & & Sig. & & 1 & 1 & 0.087 \\
\hline DHS de & Cluster 5 & 11 & -0.4538141 & & DHS de Tukey ${ }^{\mathrm{a}, \mathrm{c}}$ & Cluster 7 & 5 & -1.5369134 & & \\
\hline \multirow[t]{4}{*}{ Tukey ${ }^{a, b, c}$} & Cluster 6 & 11 & -0.3682284 & & & Cluster 6 & 11 & & -0.2790508 & \\
\hline & Cluster 7 & 5 & & 0.208895 & & Cluster 5 & 11 & & & 0.7911984 \\
\hline & Cluster 2 & 2 & & 0.3759092 & & Cluster 2 & 2 & & & 1.1697297 \\
\hline & Sig. & & 0.971 & 0.824 & & Sig. & & 1 & 1 & 0.305 \\
\hline Waller- & Cluster 5 & 11 & -0.4538141 & & Waller-Duncan ${ }^{\mathrm{a}, \mathrm{d}}$ & Cluster 7 & 5 & -1.5369134 & & \\
\hline \multirow[t]{3}{*}{ Duncan $^{\mathrm{a}, \mathrm{b}, \mathrm{d}}$} & Cluster 6 & 11 & -0.3682284 & & & Cluster 6 & 11 & & -0.2790508 & \\
\hline & Cluster 7 & 5 & & 0.208895 & & Cluster 5 & 11 & & & 0.7911984 \\
\hline & Cluster 2 & 2 & & 0.3759092 & & Cluster 2 & 2 & & & 1.1697297 \\
\hline \multicolumn{5}{|c|}{$\begin{array}{l}\text { Se muestran las medias de los grupos de subconjuntos homogéneos. } \\
\text { Basadas en las medias observadas. El término de error es la media } \\
\text { cuadrática(Error) }=.085 \text {. }\end{array}$} & \multicolumn{6}{|c|}{$\begin{array}{l}\text { Se muestran las medias de los grupos de subconjuntos homogéneos. } \\
\text { Basadas en las medias observadas. El término de error es la media } \\
\text { cuadrática(Error) }=.102 \text {. }\end{array}$} \\
\hline
\end{tabular}

Fuente: Elaboración propia (SPSS 21).

a Usa el tamaño muestral de la media armónica $=4.536$

b Los tamaños de los grupos son distintos. Se empleará la media armónica de los tamaños de los grupos. No se garantizan los niveles de error tipo I.

c Alfa $=.05$.

d Razón de seriedad del error de tipo 1/tipo $2=100$ 


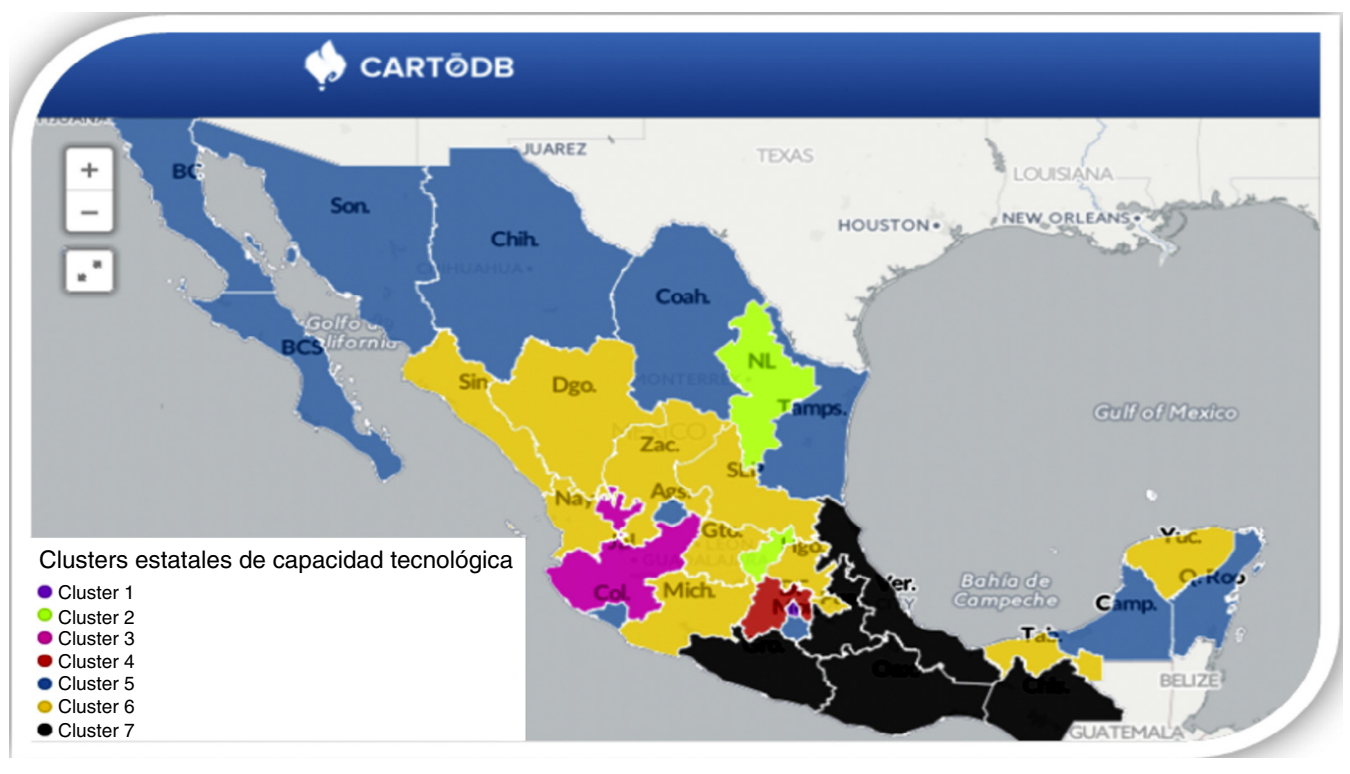

Figura 3. Clusters estatales de capacidad tecnológica en México 2012.

Fuente: elaboración propia con CARTO DB; véase también en: http://cdb.io/1tJkRKm.

- Sin embargo, no se presentan diferencias significativas entre el cluster 5 y el cluster 6 , debido a que la Sig. $>0.05$, en este caso $0.66>0.05$. En el diagrama de dispersión (fig. 2) se puede observar cierta coincidencia. Lo mismo sucede entre el cluster 7 y el cluster 2.

Se visualizan también los resultados de las pruebas post-hoc para el factor 2, definido como las capacidades tecnológicas de infraestructura, denotando que existe una diferencia estadísticamente significativa entre todos los clusters, con excepción del cluster 5 y el cluster 2 , debido a que la Sig. $>0.05$, en este caso $0.08>0.05$.

Finalmente, se puede decir que las pruebas post-hoc han mostrado diferencias significativas en la mayoría de los casos entre los grupos considerados, y a nivel conjunto (como se comprueba en los resultados del ANOVA y el gráfico de dispersión) existen diferencias significativas entre los 7 clusters. Por lo tanto, el análisis de conglomerados realizado para ambos periodos es aceptable.

\section{Conclusiones}

Este trabajo muestra una investigación empírica sobre las diferencias entre las entidades de la república mexicana en lo referente a su capacidad tecnológica y su evolución a lo largo de un período de 6 años (2006-2012). Los resultados muestran la existencia de 7 grupos de estados caracterizados claramente por distintos niveles de capacidad tecnológica; en la figura 3 se observa el predominio de los clusters 5, 6 y 7, dejando ver que los clusters del 1 al 4 manifiestan resultados satisfactorios en alguno de los factores analizados (capacidad de absorción e innovación y/o capacidad tecnológica de infraestructura); sin embargo, dichos clusters son los menos numerosos (5 entidades). Se visualiza la necesidad de descentralizar las actividades científicas y tecnológicas, a fin de contribuir a potenciar el desarrollo económico y el bienestar en todas las regiones del país, considerando su vocación productiva. 
La clasificación de las entidades en 7 clusters diferentes y el análisis de su evolución a lo largo de 6 años permiten concluir que, en general, la brecha en capacidad tecnológica $-\mathrm{y}$ por consecuencia en innovación tecnológica- entre los estados de la república mexicana es amplia y se concentra en unas pocas entidades (D.F., Nuevo León, Querétaro, Jalisco, Estado de México). Del 2006 al 2012, únicamente 3 entidades (Querétaro, Morelos y Campeche) lograron mudarse a un cluster más avanzado. Destaca la situación de Querétaro, que en el año 2006 se encontraba en el cluster 6 y para el año 2012 se ubica en el segundo cluster, acompañando a Nuevo León y solo por debajo del D.F. Esto habla de que, a pesar de que la capacidad tecnológica - y por ende la innovación- es un proceso gradual y acumulativo, existe la posibilidad de que en un periodo de 6 años los estados sí puedan avanzar considerablemente en materia de su capacidad tecnológica. El ejemplo lo han puesto estas 3 entidades, tanto en lo relativo a su capacidad de absorción e innovación como es su capacidad tecnológica de infraestructura.

Por otro lado, se pueden señalar algunas implicaciones. La primera hace referencia a la desigual distribución de la capacidad tecnológica entre los clusters observados. La combinación de los 2 factores de la capacidad tecnológica (capacidad de absorción e innovación y capacidad tecnológica de infraestructura) desempeña un importante papel a la hora de posicionar cada sistema estatal de innovación. Cada estado debería no solo observar e intentar imitar a aquellos que están en una posición o un cluster más avanzado, sino que además debería trazar su propio itinerario partiendo de su capacidad tecnológica existente, intentando tener éxito en la mejora de su posición (García, Bajo y Blázquez, 2012).

La segunda implicación se refiere a la evolución general de los sistemas de innovación. En este sentido, se debería analizar cuántos estados han mejorado y se han movido hacia un cluster superior y cuántos han retrocedido. Como se observó, ningún estado ha retrocedido a un cluster inferior, sino que algunos estados se han movido a un cluster superior. En concreto, 2 estados se han movido del cluster 6 al cluster 5 (Morelos y Campeche), un estado se ha movido del cluster 6 al cluster 2 (Querétaro) y 29 estados se han quedado más o menos como estaban. En general las variaciones en sus posiciones durante los 6 años considerados no han sido demasiado importantes, hecho que puede deberse a que las capacidades tecnológicas son generadas y destruidas lentamente a lo largo del tiempo, incluso en períodos de rápido crecimiento económico (Archibugi y Castellacci, 2008). También cabe señalar que se han reducido distancias entre el cluster 6 y el 5 en lo referente a la capacidad tecnológica de infraestructura; lo mismo sucede entre el cluster 5 y el2. Sin embargo, en los cuadrantes positivos en general solo se encuentran posicionados claramente 4 clusters (5 estados), lo cual parece coincidir con la dinámica global de la tecnología en el mundo, en la que unos pocos países generan la mayor parte del conocimiento (Archibugi y Pianta, 1996). En el largo plazo, lo anterior sugiere que México está evolucionando hacia una región en la que un pequeño grupo de estados —en torno al 16\% — domina el paisaje tecnológico, frente al $84 \%$ restante de los estados, que estarían más retrasados.

A continuación se presentan con más detalle las principales conclusiones, en términos de posibles áreas de actuación para cada uno de los grupos identificados en el análisis empírico.

Con respecto a los estados situados en los clusters 6 y 7 , estos son los que están situados en las peores posiciones en capacidad tecnológica (absorción e innovación) y tienen, por tanto, la necesidad de una clara actuación para impulsar la innovación. Con objeto de reducir su diferencia tecnológica podrían incrementar sus inversiones en $\mathrm{I}+\mathrm{D}$, ya que una mayor capacidad pública y privada de inversión en I+D podría ayudar a la industria a desarrollar sus intereses de investigación, aunado a iniciativas específicas destinadas a aumentar la cultura emprendedora, y la 
innovación en las PYMES también podrían fomentar la innovación (García, Blázquez y López 2012).

En el cluster 7, Puebla y Veracruz podrían incrementar sus esfuerzos, principalmente en materia de capacidad tecnológica de infraestructura. Los estados integrados en los clusters 6 y 7 deben hacer un esfuerzo adicional por mejorar las infraestructuras tecnológicas, ya que tienen una capacidad de absorción muy baja. En cuanto al cluster 5, se observa una posición positiva en lo referente a las capacidades tecnológicas de infraestructura; sin embargo, sus esfuerzos deberían ir encaminados a incrementar su capacidad de absorción e innovación. Los clusters 3 y 4 mantienen una posición competitiva en cuanto a las capacidades de absorción e innovación, y a pesar de ello manifiestan ciertas deficiencias en lo relativo a su capacidad tecnológica de infraestructura, por lo que esta última debería ser reforzada prioritariamente. El cluster 2, integrado por Nuevo León y Querétaro, es el mejor posicionado en capacidad tecnológica de infraestructura, y en lo referente a su capacidad de absorción e innovación se encuentra dentro del cuadrante positivo. Las empresas de los estados que lo integran determinan la competitividad tecnológica de la región. Estos estados deben prestar una particular atención a desarrollar estrategias que favorezcan la creación y difusión de conocimiento. Para ello, podrían mejorar la calidad de las instituciones de investigación, potenciar la colaboración entre las universidades y las empresas, y mejorar las leyes relacionadas con las tecnologías de la información y la comunicación, facilitando así la interacción entre todos los agentes del sistema de innovación y proporcionando la colaboración con otros actores extranjeros que les ayuden a crear nuevo conocimiento (Clarysse y Muldur, 2001, citado por García et al., 2012c). En todo caso, todavía les queda un largo recorrido para converger con el cluster 1 en lo referente a los 2 componentes analizados.

Finalmente, respecto al cluster 1, este es el más avanzado en capacidad de absorción e innovación, y se encuentra formado únicamente por el Distrito Federal. En este sentido, las entidades bien posicionadas deben tratar de atraer nuevo talento, facilitando así la creación y difusión de conocimiento de primer nivel (Archibugi y Coco, 2004). Este grupo podría representar un punto de referencia para grupos menos avanzados con objeto de que pudieran identificar actuaciones encaminadas a mejorar la innovación y que les pudieran ayudar a migrar a un cluster superior.

Las principales contribuciones de este trabajo son las siguientes:

Desde el punto de vista de la literatura sobre capacidades tecnológicas, estos resultados aportan nueva evidencia empírica sobre la existencia de 7 grupos diferentes de estados en la república mexicana en cuanto a su capacidad tecnológica, indicando las dimensiones en las que cada grupo se diferencia de los demás. Además, se muestra claramente que las diferencias entre estados están bien reflejadas por los 2 factores, como indican los resultados del análisis factorial y del análisis cluster. La presente investigación y clasificación podría ayudar a identificar y comprender los retos y oportunidades que los estados de cada cluster tienen que enfrentar en el futuro.

A pesar de las contribuciones de este estudio empírico, es preciso señalar sus limitaciones. No cabe duda de que la literatura sobre cambio tecnológico necesita continuar avanzando para encontrar mejores instrumentos de medida. En este sentido, los indicadores contemplados en este trabajo podrían reforzarse mediante la utilización de métodos de triangulación, como la utilización de índices sintéticos, o combinándolos con otros indicadores elaborados por diferentes organismos o instituciones, tal como aconseja García et al. (2012c). Además se señala como crítico el disponer de datos estadísticos estratificados a nivel estatal, debido a que se percibe que en la situación actual el subsistema de generación de estadísticas sobre ciencia y tecnología en México es limitado y denota áreas de oportunidad para su mejora.

Finalmente, cabe resaltar la necesidad de realizar estudios en profundidad de cada estado para poder proponer políticas tecnológicas diferenciadas. Lo que el presente trabajo pretende es 
simplemente señalar posibles áreas de actuación en cada estado en función de los posicionamientos obtenidos y la evolución observada de cada uno de ellos. La identificación de las áreas débiles de cada entidad en cuanto a capacidad tecnológica sugiere posibles caminos o rutas para su mejora, que obviamente deben ser complementados con estudios en profundidad de cada caso.

\section{Reflexión}

El presente trabajo, es pues, un esfuerzo por incidir en el análisis particular de la CTI pero desde una perspectiva que permita identificar propiamente las capacidades tecnológicas a nivel estado, aglomerando a las entidades que comparten condiciones similares en dicha materia y poder, de esta forma, contar con un mapa que dibuje las distancias y el camino por recorrer, de un cluster a otro, en aras del progreso y la competitividad y con el objetivo, también, que este tipo de estudios sean fuente de referencia para incurrir en políticas tecnológicas diferenciadas y acordes a las necesidades relativas a cada grupo de estados.

Decir que México no crece es olvidar que en nuestro país hay estados que durante ciertos periodos bien podrían ser clasificados como tigres asiáticos, conviviendo con entidades que sufren crisis económicas de proporciones similares a la griega. Por lo tanto, el problema no es que México no crezca, sino que crece a 32 pasos diferentes (Ríos, 2014).

Los resultados de esta investigación sugieren una analogía similar. México sí hace uso efectivo del conocimiento tecnológico para cambiar las tecnologías existentes y desarrollar nuevos productos y procesos, solo que dicha capacidad avanza a 7 pasos diferentes ( 7 clusters estatales de capacidad tecnológica). Por lo tanto, para incrementar dichas capacidades es necesario un ejercicio de benchmarking, tomando como punto de referencia aquellos estados que están realizando un buen trabajo en materia de promoción de la innovación otorgando las condiciones proclives para ejecutarla. Fijarnos en el D.F., Nuevo León y Querétaro ${ }^{5}$ como casos de éxito quizá sea más provechoso que voltear la vista a Suiza, Reino Unido o Suecia ${ }^{6}$, dado que los primeros forman parte de nuestro contexto. Sin embargo, para la observancia de los primeros se requiere de diversos tipos y metodologías de investigación para analizar el tema.

Los hallazgos de la presente investigación enriquecen el debate y la discusión relativa a las capacidades de innovación tecnológica en México. Algunos de los resultados son convergentes con estudios previos; por ejemplo, el top 3 de estados con mayores capacidades de innovación y capacidades tecnológicas de infraestructura coincide con el top 3 presentado en el ranking nacional de ciencia, tecnología e innovación (FCCyT, 2014). Sin embargo, dado que la metodología y el enfoque de investigación son diferentes, se aprecia que la estratificación de estos 7 clusters marca la pauta para reflexionar como generalmente se suele estratificar la innovación en 3 grupos $^{7}$. No obstante, los resultados de la presente investigación sugieren la existencia de 4 grupos de estados (casi aislados) que muestran una dinámica eficiente y claramente diferente en materia de capacidad tecnológica en comparación con el resto de la república.

Resulta preocupante la situación principalmente de Chiapas, Guerrero y Oaxaca. Por su parte, los clusters 5 y 6 se ubican también en los cuadrantes negativos, y las entidades que se congregan

\footnotetext{
${ }^{5}$ El D.F., en nuestro estudio, resultó la entidad puntera en capacidades tecnológicas, mientras que Nuevo León y Querétaro pertenecen al segundo cluster.

${ }^{6}$ Países de mejor ranking según WIPO (2014).

7 Clusters A, B, C en el ranking nacional de ciencia tecnología e innovación (FCCyT, 2014); estados en construcción, estados en transición y estados en consolidación según el PECITI, 2014-2018.
} 
en dichos clusters son consideradas estados en transición en inversión de CTI y economía del conocimiento (PECITI, 2014). Finalmente, los clusters del 1 al 4 presentan mejores condiciones en cuanto a sus capacidades tecnológicas; en este caso, los estados en consolidación resultaron únicamente D.F., Nuevo León, Querétaro y Jalisco. Derivado de ello, surge la interrogante de ¿por qué parece que la capacidad tecnológica se concentra en unos cuantos estados? ¿En qué medida esto depende de la ubicación? ¿Cómo se relaciona cada cluster con el desarrollo económico? ¿Cómo la creación y la acumulación de capacidades tecnológicas estatales impactan tanto en el nivel de vida como en la calidad de vida de los mexicanos? Preguntas que se abren como sugerencias de investigación y que podrían ser respondidas bajo diversas metodologías. Finalmente, se auguran buenas expectativas: según el PECITI (2014), se tiene planteado que GIDE/PIB para el año 2018 sea del 1\%, cifra que impulsaría las capacidades de ciencia, tecnología e innovación. Sin embargo, una tarea pendiente es revertir que se privilegie el pensamiento mágico sobre el lógico científico, ya que, según la Encuesta sobre la Percepción Pública de la Ciencia y la Tecnología en México (Enpecyt, 2011), el 57.5\% de los mexicanos considera que, debido a sus conocimientos, «los investigadores y científicos tienen un poder que los hace peligrosos». Para que los incentivos en materia de ciencia y tecnología funcionen debería cambiar dicha percepción, que repliegue en el aumento de nuestro acervo de recursos humanos dedicados a la investigación, ciencia y tecnología.

\section{Referencias}

Álvarez, R. (1995). Estadística multivalente y no paramétrica con SPSS. Madrid: Díaz de Santos.

Archibugi, D. (1988). In search of a useful measure of technological innovation (to make economists happy without discontenting technologists). Technological Forecasting and Social Change, 34, 253-277.

Archibugi, D. y Castellacci, F. (2008). The technology clubs: The distribution of knowledge across nations. Research Policy, 37, 1659-1673.

Archibugi, D. y Coco, A. (2004). A new indicator of technological capabilities for developed and developing countries (ArCo). World Development, 32, 629-654.

Archibugi, D. y Coco, A. (2005). Measuring technological capabilities at the country level: A survey and a menu for choice. Research Policy, 34, 175-194.

Archibugi, D. y Pianta, M. (1996). Measuring technological change through patents and innovation surveys. Technovation, 16, 451-468.

Biggs, T., Shah, M. y Srivastava, P. (1995). Technological capabilities and learning in African enterprises. World Bank technical paper; no. WTP 288. Africa Technical Department series. Washington, D.C.: The World Bank. http://documents. worldbank.org/curated/en/649901468773714947/Technological-capabilities-and-learning-in-African-enterprises

Blázquez de le Hera, M. L. y García-Ochoa Mayor, M. (2009). Clusters de innovación tecnológica en Latinoamérica. GCG Georgetown University - Universia, 3, 16-33.

Borrastero, C. (2012). Notas sobre la cuestión del excedente en las teorías evolucionistas neoschumpeterianas. Papeles de trabajo. Revista electrónica del Instituto de Altos Estudios Sociales de la Universidad Nacional de General San Martín, 100-126.

Brunner, J. (2011). Chile: informe sobre capacidades tecnológicas. pp. 1-41. Instituto de Economía Política.

Calderón, M. y Hartmann, D. (2010). Una revisión del pensamiento evolucionista y el enfoque de los sistemas de innovación. RUDICS, 1-18.

Cepal. (2007). Serie Estudios y Perspectivas. Indicadores de capacidades tecnológicas en América Latina. México: Naciones Unidas [consultado 10 Ene 2015]. Disponible en: http://www.cepal.org/es/publicaciones/5014-indicadoresde-capacidades-tecnologicas-en-america-latina.

Chinaprayoon, C. (2007). Science, Technology and Innovation Composite Indicators for Developing Countries. Georgia Institute of Tecnology.

Close, E. y Garita, M. (2011). La innovación en las empresas de Guatemala. ECO Revista Académica., 27-36.

Diaconu, M. (2011). Technological innovation: Concept, process, typology and implications in the economy. Theoretical \& Applied Economics., 18(10), 127-144. 
Dosi, G. (2008). La interpretación evolucionista de las dinámicas soio económicas. En R. Viale (Ed.), Las nuevas economías. De la economía evolucionista a la economía cognitiva: Más allá de las fallas de la teoría neoclásica (pp. 29-44). México: Flacso México.

Dutrénit, G., Capdeville, M., Corona, J., Puchet, M. y Vera-cruz, F. (2010). El Sistema Nacional de Innovación Mexicano. $U A M, 1-62$.

Enpecyt. (2011). Encuesta sobre la Percepción Pública de la Ciencia y la Tecnología en México. INEGI.

FCCyT. (2014). Ranking Nacional de Ciencia Tecnología e Innovación. Capacidades y Oportunidades de los sistemas estatales de CTI. México: FCCyT.

García, M., Blázquez, M. y Ruiz, E. (2012). Empirical study of national technological innovation capability in Africa. SAJEMS, 440-463.

García-Ochoa Mayor, M., Bajo Davó, N. y Blázquez de la Hera, M. L. (2012). La innovación tecnológica como variable determinante en la competitividad de los países. Revista de Economía Mundial, 31, 137-166.

García-Ochoa Mayor, M., Blázquez de la Hera, M. L. y López Sánchez, J. I. (2012). Uso y aplicación de la técnica de análisis estadístico multivariante de cluster sobre la capacidad de innovación tecnológica en Latinoamérica y España. Innovar, 22, 21-40.

Guzmán, D. (2008). Las capacidades tecnológicas en la industria del calzado en México. México: I.P.N.

Martín, Q., Cabero, M. y de Paz, Y. (2008). Tratamiento estadístico de datos con SPSS. España: Thomson.

Morales, M. (2009). Teoría Económica Evolutiva de la Empresa: ¿una alternativa a la teoría neoclásica? Revista Latinoamericana de Economía, 161-183.

PECITI. (2014). Programa Especial de Ciencia, Tecnología e Innovación 2014-2018. CONACyT.

Pedroza, H. (2006). Sistema de análisis estadístico con SPSS. Nicaragua: INTA.

Quiñones A, Tezanos S (2011) Cooperación científico-tecnológica para el desarrollo internacional: una apuesta desde la innovación [consultado 10 Ene 2015]. Disponible en: http://www.ciberoamericana.com/pdf/DT_2011_02.pdf.

Ríos, V. (2014). México sí crece, solo que a 32 pasos diferentes. Excelsior.

Schumpeter, J. (2005). Development. Journal of Economic Literature, 108-120.

Stern, S., Porter, M. E. y Furman, J. L. (2000). The Determinants of National Innovative Capability. NBER Working Paper Series. http://dx.doi.org/10.3386/w7876

Velarde, E., Garza, E. y Coronado, E. (2011). El desarrollo de capacidades tecnológicas y la vinculación con instituciones educativas. Global Conference on Business and Finance Proceedings, 6(2), 1-7.

WIPO (2014). The Global Innovation Index 2014. INSEAD [consultado 10 Ene 2015]. Disponible en: https://www.globalinnovationindex.org/userfiles/file/reportpdf/gii-2014-v5.pdf. 